\title{
Defining New Pancreatic Tumour Entities by Molecular Analysis
}

\author{
A.L. Mihaljevic $^{a} \quad$ I. Esposito ${ }^{b, c}$ C.W. Michalski ${ }^{a} \quad$ J. Kleeffa $\quad$ H. Friess ${ }^{a}$ \\ aDepartment of Surgery, Klinikum rechts der Isar, Technische Universität München, ${ }^{\mathrm{b}}$ Institute of Pathology, \\ Technische Universität München, and 'Institute of Pathology, Helmholtz Zentrum München, Munich, Germany
}

\section{Key Words}

Pancreatic cancer - Tumour entities - Molecular analysis • Mutation

\begin{abstract}
Recent advances in molecular biology, biochemistry and genetics have broadened our understanding of tumourigenesis and of the maintenance and spread of pancreatic cancer far beyond traditional microscopic histopathological analysis. While the main focus of pancreatic cancer research has been on pancreatic ductal adenocarcinoma, molecular research has also led to a better understanding of rare tumours of the pancreas, as well as to the definition of previously unknown tumour entities that can only be identified through the application of molecular tools. Furthermore, molecular analysis increasingly reveals the genetic and cell biological heterogeneity of established tumour entities, making subclassification of tumours possible. Genetic and molecular approaches may, therefore, not only lead to a better understanding of the pathogenesis of pancreatic tumours, but also culminate in more precise diagnosis as well as individually tailored treatment strategies for affected patients.
\end{abstract}

Copyright $\odot 2009$ S. Karger AG, Basel and IAP

\section{Introduction}

The pancreas gives rise to a variety of different neoplasms with diverse origins, differentiation and biological behaviour [1]. Traditionally, pancreatic tumours have been defined using histopathological features, i.e., morphological characteristics only. The spectrum of histopathological entities has grown rapidly with the widespread and standardized use of immunohistochemistry. The advent of standardized molecular biological tools has, however, triggered a surge in the investigation of pancreatic tumours on a molecular level to elucidate the mechanisms of tumourigenesis, local recurrence and metastasis in order to identify new therapeutic targets. This has led to the identification of frequent molecular alterations typical of certain tumour entities, and these alterations are increasingly used to classify or subclassify pancreatic tumours.

\section{Genetic Characteristics of Pancreatic Tumours}

Genetic changes are analysed either on a chromosomal level, usually by determining loss of heterozygosity, or on a gene level, by amplification and sequencing. Microarray techniques are increasingly applied to study changes of transcriptional expression in pancreatic tumours as

\section{KARGER \\ Fax +4161306 1234 \\ E-Mail karger@karger.ch}

www.karger.com
(C) 2009 S. Karger AG, Basel and IAP

$1424-3903 / 09 / 0094-0334 \$ 26.00 / 0$

Accessible online at:

www.karger.com/pan
J. Kleeff

Chirurgische Klinik und Poliklinik, Klinikum rechts der Isar

Technische Universität München, Ismaningerstrasse 22

DE-81675 Munich (Germany)

Tel. +49 894140 5098, Fax +49894140 4870, E-Mail kleeff@chir.med.tu-muenchen.de 
Table 1. Frequent genetic alterations in pancreatic tumours

\begin{tabular}{|c|c|c|c|}
\hline Tumour entity & Genetic alteration & Frequency & Ref. \\
\hline \multirow[t]{6}{*}{$P D A C$} & K-ras & $75-100 \%$ & 2,44 \\
\hline & INK4A (p16, CDKN2A) & $40-100 \%$ & 8,45 \\
\hline & TP53 & $40-87 \%$ & $6,7,9$ \\
\hline & DPC4/SMAD4/MADH4 & $55-66 \%$ & 9,10 \\
\hline & INK4B (p15, CDKN2B) & $27-48 \%$ & 2 \\
\hline & FHIT & $66-70 \%$ & 2 \\
\hline \multicolumn{4}{|l|}{ PET } \\
\hline \multirow[t]{6}{*}{ Non-functional } & $\mathrm{LOH}(6 \mathrm{q}, 11 \mathrm{q})$ & $>60 \%$ & 46 \\
\hline & LOH $(11 p, 21 q)$ & $50 \%$ & 46 \\
\hline & $\mathrm{LOH}(3 \mathrm{p})$ & $45 \%$ & 43 \\
\hline & LOH (p16/MTSI) & $43 \%$ & \\
\hline & $\mathrm{LOH}(\mathrm{MEN} 1)$ & $55 \%$ & \\
\hline & $\mathrm{LOH}(\mathrm{p} 53)$ & $50 \%$ & \\
\hline \multicolumn{4}{|l|}{ Functional $^{1}$} \\
\hline \multirow[t]{3}{*}{ Insulinoma } & LOH (MEN 1) & $22 \%$ & 43 \\
\hline & $12 p($ Ras $)$ & $21 \%$ & \\
\hline & LOH (MADH4/SMAD4) & $27 \%$ & \\
\hline \multirow[t]{3}{*}{ Glucagonoma } & $\mathrm{LOH}(3 \mathrm{p})$ & $71 \%$ & \\
\hline & LOH (MEN 1) & $80 \%$ & \\
\hline & LOH (MADH4/SMAD4) & $60 \%$ & \\
\hline \multirow[t]{2}{*}{ Acinar cell CA } & LOH 11p & $50 \%$ & 47 \\
\hline & APC & $23 \%$ & 47 \\
\hline \multirow{5}{*}{$\begin{array}{l}\text { Cystic tumours } \\
\text { IPMN }\end{array}$} & & & \\
\hline & K-ras & $\begin{array}{l}0 \% \text { normal } \\
75 \% \text { high-grade dysplasia }\end{array}$ & 17,18 \\
\hline & DPC4/SMAD4/MADH4 & & 19 \\
\hline & STK1/LKB1 & & 20 \\
\hline & P13KCA & & 21 \\
\hline \multirow[t]{6}{*}{$\mathrm{MCN}^{2}$} & K-ras & $89 \%$ carcinoma & 13 \\
\hline & & $20 \%$ adenoma & \\
\hline & TP53 & $44 \%$ carcinoma & 13 \\
\hline & & $0 \%$ adenoma & \\
\hline & DPC4/SMAD4/MADH4 & $14 \%$ carcinoma & 14 \\
\hline & & $100 \%$ adenoma & \\
\hline SCN & VHL & $40 \%$ & 24,25 \\
\hline SPT & $\beta$-Catenin & $>90 \%$ & 26 \\
\hline
\end{tabular}

Only mutations with a high frequency are listed. PDAC = Pancreatic ductal adenocarcinoma; $\mathrm{PET}$ = pancreatic endocrine tumour; $\mathrm{MCN}$ = mucinous cystic neoplasm; IPMN = intraductal papillary mucinous neoplasm; SPT = solid-pseudopapillary tumour; $\mathrm{LOH}=$ loss of heterozygosity.

${ }^{1}$ Only limited data is available on other hormone-producing PETs other than insulinomas and glucagonomas [43].

${ }^{2}$ Frequency depends on invasiveness of the tumour, percentage given for tumours with invasive component. compared to normal pancreatic tissue. Characteristic genetic alterations of some pancreatic tumours are listed in table 1.

In the case of pancreatic ductal adenocarcinoma (PDAC), the most frequent tumour of the pancreas, a number of these alterations have been identified and linked to a tumour progression model beginning with intraductal epithelial proliferations (pancreatic intraepithelial neoplasia (PanIN)) and developing into invasive pancreatic cancer [reviewed in 2,3]. Mutations of the K-ras 
oncogene are found in $75-100 \%$ of PDAC, making it the tumour with the highest incidence of K-ras mutations [4]. A mutation in codon 12 of the K-ras oncogene accounts for a constitutively activated form of the small GTPase, which is believed to be an early event in PDAC development [3]. The tumour suppressor gene TP53, encoding the transcription factor p53, on the other hand, is affected in more than $50 \%$ of cases by missense mutations in its DNA binding domain, accompanied by allelic loss of the wildtype gene [5-7]. Homozygous deletion of the p16INK4A/ p14ARF locus encoding the tumour suppressors INK4A/ p16 and ARF can be observed in $80-95 \%$ of PDAC, and occurs at a later stage of tumour progression [8]. Finally, the transcription factor MADH4/SMAD4 is inactivated by deletion or mutation in over $50 \%$ of cases $[9,10]$.

There is growing interest in cystic tumours of the pancreas since at least two types - mucinous cystic neoplasms $(\mathrm{MCN})$ and intraductal papillary mucinous neoplasms (IPMN) - can be precursor lesions of PDAC [11]. MCN are cystic mucin-producing lesions with a distinctive cellrich ovarian-type stroma. MCN typically occur in women and show an invasive component in approximately one third of cases [12]. Like PanIN, invasive MCN show a molecular progression that mirrors histological progression from adenoma to carcinoma. The same genes known to be involved in PanIN progression also play a role in MCN tumour progression. K-ras mutations are detected in $20 \%$ of MCN adenomas, but feature in $89 \%$ of MCN with a carcinoma component [13], while inactivation of TP53 and MADH4/SMAD4 is found almost exclusively in invasive components $[13,14]$. Furthermore, molecular analysis has revealed that the ovarian-like stroma of MCN is hormone-sensitive [11], and a global expression analysis of MCN has revealed an ovary-like expression programme within the stroma [15].

IPMN are intraductal, mucin-producing neoplasms that are classified into main-duct and branch-duct types depending on their localization within the duct system. These often multifocal lesions are classified depending on the epithelial lining into 'intestinal', 'pancreaticobiliary' (mostly main duct IPMN) and 'gastric foveolar' (often branch-duct IPMN) types [1]. These histological subtypes correlate with differential expression of apomucins: intestinal type IPMN express MUC2 and MUC5AC but not MUC1, while pancreaticobiliary IPMN typically express MUC1 and MUC5AC. Gastric foveolar types solely express MUC5AC, but not MUC1 or 2 [16]. Molecular subclassification is important in IPMN since different types show markedly different malignancy patterns and prognoses [11]. Like PanIN and MCN, IPMN exhibit a multistep progression from benign to malignant lesions. Again, K-ras mutation is an early step in IPMN tumourigenesis $[17,18]$, but in contrary to PanIN, MADH4/ SMAD4/PDC4 gene inactivation is uncommon in IPMN and is only seen in invasive components [19]. However, inactivation of the Peutz-Jeghers syndrome gene STK1/ LKB1 is seen in approximately $30 \%$ of IPMN [20]. Further genetic alterations frequently found in IPMN are activating mutations of the P13KCA, a member of the Akt signalling pathway [21]. An example of increasing subclassification of pancreatic tumours with molecular methods is the intraductal oncocytic papillary neoplasm (IOPN), which is regarded as a subtype of IPMN based on histopathologic features. Molecular findings suggest that IOPN may be different from IPMN [42]. In particular, IOPN lack the K-ras gene mutation and show alternate MUC expression [22].

Serous cystic neoplasms (SCN) are microcystic tumours that predominantly affect female patients. SCN are presumed to arise from the centroacinar duct system, and express MUC6 as well as Glut-1 [22, 23]. Sporadic SCN show von Hippel-Lindau (VHL) gene alterations in up to $40 \%$ of cases [24,25], implicating VHL in the tumourigenesis of SCN.

While the histopathological characteristics of solidpseudopapillary neoplasms (SPN) - rare, sometimes cystic tumours of the pancreas almost exclusively found in young women - are well described, the origin of SPN tumour cells remains enigmatic, since they lack evidence of ductal, acinar or frank endocrine differentiation. The most typical features of SPN can therefore only be found on a molecular level: namely, the expression of CD10 and cyclin D1 and point mutations in the $\beta$-catenin gene, which can be demonstrated in over $90 \%$ of the cases [22, 26]. Further molecular characterization of SPT is warranted to elucidate their genetic origin.

\section{Epigenetic Changes in Pancreatic Tumours}

A large number of epigenetic alterations have been identified in pancreatic cancer as causing differential gene expression [27-35]. Analysis of epigenetic modifications of genes involved in pancreatic tumour development may be used to subclassify tumour entities and may also predict biological tumour behaviour. For example, Sato et al. [27] showed that the extracellular matrix protein SPARC/osteonectin, which influences cellular differentiation, proliferation and angiogenesis [36], is a frequent target of DNA methylation in PDAC. 
Fig. 1. Cluster analysis for transcriptional profiling of an unknown tumour entity (III primary; IV liver metastasis; $\mathrm{V}$ abdominal metastasis) compared to the profiles of normal pancreatic tissue (I) and PDAC (II). In the resulting bi-plot, each hybridization of an individual sample is depicted as a coloured square. Genes that exhibited significantly differential transcription levels are shown as grey dots. The closer the co-localization of two spots (both genes and tumours), the higher is the degree of association between them. As a control, normal pancreatic tissue was used (cluster I, red circle). As all other experiments were compared to this control, only the centre of gravity of all normal tissue samples is shown rather than individual experiments. Reprinted with permission (conferred through the 'Copyright Clearance Center') from Loos et al. [41].

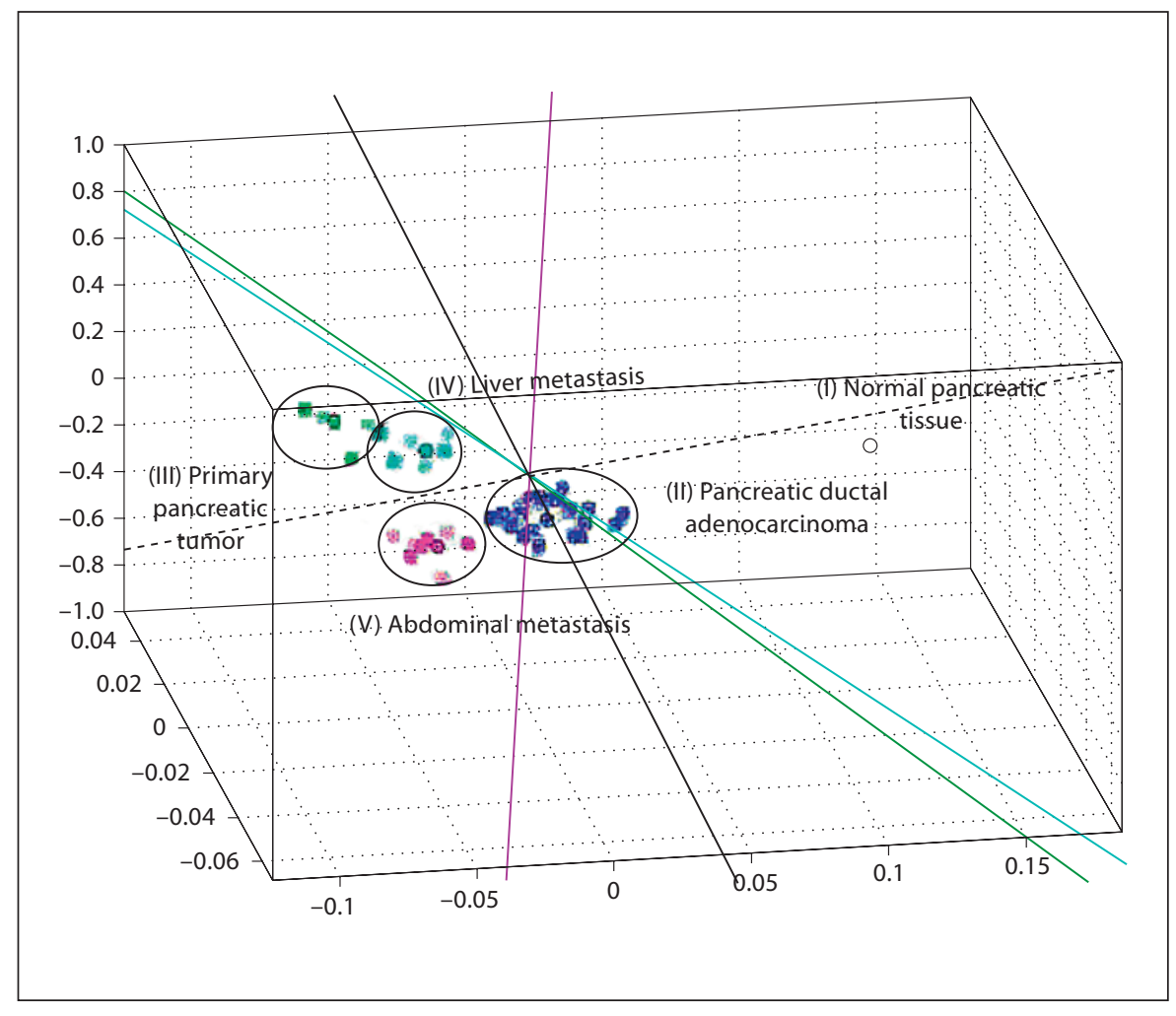

\section{Cell Biological Characteristics of Pancreatic Tumours}

Elucidating alterations on the DNA level is only one aspect of molecular analysis. Research has focused even more on changes in the expression levels of proteins in pancreatic tumour cells, since the biological behaviour of tumour cells depends on the complex interactions of proteins within the cancer cells themselves and of proteinprotein interactions between cells. Deregulation of expression has been found in multiple proteins, especially in growth-promoting or growth-inhibiting signalling pathways [reviewed in 2, 3, 37]. The listing of the number of overexpressed oncogenes or of the suppressed tumour suppressor genes as well as of the sheer number of analysed genes is far beyond the scope of this article. Recently, a freely accessible database (www.pancreasexpression. org) collecting all known analysed proteins and genes involved in pancreatic tumour development was made available (see article by $\mathrm{C}$. Chelala et al., this issue).

The wealth of information that can be gained by expressional analysis of pancreatic tumours will help to further subclassify tumour entities, leading the way to individually tailored diagnosis and treatment options.

\section{Examples of New Tumour Entities Defined by Molecular Analysis}

The usefulness of molecular tools in defining new tumour entities is demonstrated in a recent study [38] that reports an unusual microcystic tumour of the pancreas histologically characterized by a tubulopapillary architecture and an expansive growth pattern. This tumour could not be classified on the base of conventional histopathological or standard immunhistochemical characteristics. In order to elucidate its origin, the authors checked for molecular alterations that are commonly found in other pancreatic tumours (see table 1), such as K-ras, p16, p53, DPC4 and $\beta$-catenin by immunhistochemistry and gene sequencing. Furthermore, transcriptional profiling by microarray analysis was employed to find differentially transcribed genes that are up- or downregulated in the tumour when compared to samples of known tumour entities or normal tissues. The resulting cluster analysis $[39,40]$ revealed that the tumour had a distinct profile when compared with other pancreatic tumour entities. Taken together, the authors concluded that the tumour under investigation is a new hitherto unknown tumour entity. 
The validity of such an approach has been confirmed by Loos et al. [41] who defined a solid type clear cell carcinoma of the pancreas as new tumour entity by applying the methods mentioned above (fig. 1).

\section{Outlook}

Molecular tools become increasingly important for the precise diagnosis of pancreatic neoplasms and have been used to subclassify already established tumour entities. Furthermore, new tumour entities are being defined based on molecular characteristics rather than on their histopathologic appearance. The abundance of information that can be drawn from molecular analyses of gene alterations as well as protein expression patterns of indi- vidual tumours goes far beyond a mere descriptive study of tumours, and allows instead an insight into the triggering factors causing tumour development as well as the mechanism for tumour maintenance. Based on the exact tumour profile, we may be able to tailor treatment strategies to the individual patient in the future as new therapeutic options for pancreatic tumours arise.

\section{Acknowledgements}

This research was supported by the European Community's FP6 funding (MolDiag-Paca, LSHB-CT-2006-018771). This publication reflects only the authors' views. The European Community is not liable for any use that may be made of the information herein.

\section{References}

1 Solcia EC, et al: Tumors of the Pancreas. Atlas of Tumour Pathology. Washington, Armed Forces Institute of Pathology, 1997.

$\$ 2$ Li D, et al: Pancreatic cancer. Lancet 2004; 363:1049-1057.

-3 Kern SE, et al: An introduction to pancreatic adenocarcinoma genetics, pathology and therapy. Cancer Biol Ther 2002;1:607-613.

-4 Almoguera C, et al: Most human carcinomas of the exocrine pancreas contain mutant cK-ras genes. Cell 1988;53:549-554.

5 Rozenblum E, et al: Suppressive-suppressive pathways in pancreatic carcinoma. Cancer Res 1997;57:1731-1734.

-6 Boschman CR, et al: Expression of p53 protein in precursor lesions and adenocarcinoma of human pancreas. Am J Pathol 1994; 145:1291-1295.

7 DiGiuseppe JA, et al: Overexpression of p53 protein in adenocarcinoma of the pancreas. Am J Clin Pathol 1994;101:684-688.

$\checkmark 8$ Wilentz RE, et al: Inactivation of the p16 (INK4A) suppressor-suppressor gene in pancreatic duct lesions: loss of intranuclear expression. Cancer Res 1998;58:4740-4744.

-9 Biankin AV, et al: Overexpression of $\mathrm{p} 21$ (WAF1/CIP1) is an early event in the development of pancreatic intraepithelial neoplasia. Cancer Res 2001;61:8830-8837.

10 Wilentz RE, et al: Loss of expression of Dpc4 in pancreatic intraepithelial neoplasia: evidence that DPC4 inactivation occurs late in neoplastic progression. Cancer Res 2000;60: 2002-2006

11 Singh M, Maitra A: Precursor lesions of pancreatic cancer: molecular pathology and clinical implications. Pancreatology 2007;7: 9-19.
12 Klimstra DS: Cystic, mucin-producing neoplasms of the pancreas: the distinguishing features of mucinous cystic neoplasms and intraductal papillary mucinous neoplasms. Semin Diagn Pathol 2005;22:318-329.

13 Jimenez RE, et al: Sequential accumulation of K-ras mutations and p53 overexpression in the progression of pancreatic mucinous cystic neoplasms to malignancy. Ann Surg 1999;230:501-511.

14 Iacobuzio-Donahue CA, et al: Dpc4 protein in mucinous cystic neoplasms of the pancreas: frequent loss of expression in invasive carcinomas suggests a role in genetic progression. Am J Surg Pathol 2000;24:15441548

15 Fukushima N, et al: Characterization of gene expression in mucinous cystic neoplasms of the pancreas using oligonucleotide microarrays. Oncogene 2004;23:9042-9051.

16 Adsay NV: Cystic neoplasia of the pancreas: pathology and biology. J Gastrointest Surg 2008;12:401-404.

17 Kondo H, et al: Detection of K-ras gene mutations at codon 12 in the pancreatic juice of patients with intraductal papillary mucinous tumours of the pancreas. Cancer 1997; 79:900-905.

18 Yoshizawa K, et al: Clonality and k-ras mutation analyses of epithelia in intraductal papillary mucinous tumour and mucinous cystic tumour of the pancreas. Virchows Arch 2002;441:437-443.

19 Iacobuzio-Donahue CA, et al: $\mathrm{Dpc}-4$ protein is expressed in virtually all human intraductal papillary mucinous neoplasms of the pancreas: comparison with conventional ductal adenocarcinomas. Am J Pathol 2000 157:755-761.
20 Sahin F, et al: Loss of Stk11/Lkb1 expression in pancreatic and biliary neoplasms. Mod Pathol 2003;16:686-691.

21 Schonleben F, et al: PIK3CA mutations in intraductal papillary mucinous neoplasm/carcinoma of the pancreas. Clin Cancer Res 2006;12:3851-3855.

22 Adsay N: Cystic lesions of the pancreas. Mod Pathol 2007;20:71-93.

23 Singh RB, et al: The role of GLUT-1 in 'clearcell' ductal tumours of the pancreas. Mod Pathol 2006;19:280A.

24 Mohr VH, et al: Histopathology and molecular genetics of multiple cysts and microcystic (serous) adenomas of the pancreas in von Hippel-Lindau patients. Am J Pathol 2000; 157:1615-1621.

25 Kosmahl M, et al: Serous cystic neoplasms of the pancreas: an immunohistochemical analysis revealing $\alpha$-inhibin, neuron-specific enolase, and MUC6 as new markers. Am J Surg Pathol 2004;28:339-346.

26 Kosmahl M, et al: Solid-pseudopapillary tumour of the pancreas: its origin revisited. Virchows Arch 2000;436:473-480.

27 Sato N, et al: SPARC/osteonectin is a frequent target for aberrant methylation in pancreatic adenocarcinoma and a mediator of tumor-stromal interactions. Oncogene 2003; 22:5021-5030.

28 Sato N, Goggins M: Epigenetic alterations in intraductal papillary mucinous neoplasms of the pancreas. J Hepatobiliary Pancreat Surg 2006;13:280-285.

29 Sato N, et al: Epigenetic down-regulation of CDKN1C/p57KIP2 in pancreatic ductal neoplasms identified by gene expression profiling. Clin Cancer Res 2005;11:46814688 . 
30 Sato N, et al: The chemokine receptor CXCR4 is regulated by DNA methylation in pancreatic cancer. Cancer Biol Ther 2005;4:70-76.

-31 Sato N, et al: Identification of maspin and $\mathrm{S} 100 \mathrm{P}$ as novel hypomethylation targets in pancreatic cancer using global gene expression profiling. Oncogene 2004;23:15311538.

>32 Matsubayashi H, et al: Methylation of cyclin D2 is observed frequently in pancreatic cancer but is also an age-related phenomenon in gastrointestinal tissues. Clin Cancer Res 2003;9:1446-1452.

33 Fukushima N, et al: Aberrant methylation of preproenkephalin and p16 genes in pancreatic intraepithelial neoplasia and pancreatic ductal adenocarcinoma. Am J Pathol 2002; 160:1573-1581.

34 Jansen M, et al: Aberrant methylation of the $5^{\prime} \mathrm{CpG}$ island of TSLC1 is common in pancreatic ductal adenocarcinoma and is first manifest in high-grade PanlNs. Cancer Biol Ther 2002;1:293-296.

35 Ueki T, et al: Identification and characterization of differentially methylated CpG islands in pancreatic carcinoma. Cancer Res 2001; 61:8540-8546.
36 Bradshaw AD, Sage EH: SPARC, a matricellular protein that functions in cellular differentiation and tissue response to injury. J Clin Invest 2001;107:1049-1054.

37 Kleeff J, et al: Pancreatic cancer: from bench to 5-year survival. Pancreas 2006;33:111118.

38 Esposito I, et al: Microcystic tubulopapillary carcinoma of the pancreas: a new tumour entity? Virchows Arch 2004;444:447-453.

39 Beissbarth T, et al: Processing and quality control of DNA array hybridization data. Bioinformatics 2000;16:1014-1022.

40 Fellenberg K, et al: Correspondence analysis applied to microarray data. Proc Natl Acad Sci USA 2001;98:10781-10786.

41 Loos M, et al: Solid type clear cell carcinoma of the pancreas: differential diagnosis of an unusual case and review of the literature. Virchows Arch 2007;450:719-726.
42 Z'Graggen K, et al: Prevalence of activating K-ras mutations in the evolutionary stages of neoplasia in intraductal papillary mucinous tumours of the pancreas. Ann Surg 1997;226: 491-500.

43 Rindi G, Bordi C: Endocrine tumours of the gastrointestinal tract: aetiology, molecular pathogenesis and genetics. Best Pract Res Clin Gastroenterol 2005;19:519-534.

44 Schneider G, Schmid RM: Genetic alterations in pancreatic carcinoma. Mol Cancer 2003;2:15.

45 Moskaluk CA, Hruban RH, Kern SE: p16 and K-ras gene mutations in the intraductal precursors of human pancreatic adenocarcinoma. Cancer Res 1997;57:2140-2143.

46 Rigaud G, et al: High-resolution allelotype of nonfunctional pancreatic endocrine tumours: identification of two molecular subgroups with clinical implications. Cancer Res 2001;61:285-292.

-47 Abraham SC, et al: Genetic and immunohistochemical analysis of pancreatic acinar cell carcinoma: frequent allelic loss on chromosome $11 \mathrm{p}$ and alterations in the $\mathrm{APC} / \beta$ catenin pathway. Am J Pathol 2002;160:953962. 